\title{
AVALIAÇÃO DOS MÉTODOS DE IMAGEM NO DIAGNÓSTICO DA UROLITÍASE: REVISÃO DA LITERATURA*
}

\author{
Ricardo Miguel Costa de Freitas ${ }^{1}$, Luciana Costa Silva ${ }^{2}$, Jovita Lane Soares Santos ${ }^{1}$, \\ Wilson Campos Tavares Júnior ${ }^{1}$
}

\begin{abstract}
Resumo 0 objetivo deste trabalho foi comparar a tomografia computadorizada helicoidal com os métodos imaginológicos atualmente disponíveis para a abordagem dos pacientes com dor lombar aguda e revisar brevemente as características fisiopatológicas da urolitíase e a evolução da sua abordagem diagnóstica. Foi revista a literatura publicada nos últimos 30 anos, comparando os seguintes métodos: radiografia simples de abdome, urografia excretora, ultra-sonografia e tomografia computadorizada helicoidal. Esta última se mostrou método de alta sensibilidade e especificidade para o diagnóstico de urolitíase, sendo que, virtualmente, todos os cálculos são identificados por este método, exceto em pacientes em uso de indinavir. A radiografia simples de abdome associada à ultra-sonografia têm acurácia semelhante à tomografia computadorizada helicoidal, contudo, esta foi superior como método de avaliação isolado. A literatura sugere que a tomografia computadorizada helicoidal é útil na avaliação de pacientes com dor lombar aguda quando disponível, possibilitando também o diagnóstico diferencial entre as várias doenças que simulam os sintomas de urolitíase. Unitermos: Dor lombar aguda; Radiografia simples do abdome; Tomografia computadorizada helicoidal; Ultrasonografia do trato urinário; Urografia excretora; Urolitíase.
\end{abstract}

Abstract Evaluation of imaging methods in the diagnosis of urolithiasis: review of the literature.

The aim of this study is to compare helical computed tomography with imaging studies currently used to evaluate patients with acute low back pain. In addition, we briefly review the pathophysiology of urolithiasis. The literature published in the last $\mathbf{3 0}$ years was reviewed, and the following methods were discussed: plain abdominal radiographs, intravenous pielography, ultrasound of the urinary tract, and helical computed tomography. Helical computed tomography showed high sensitivity and specificity for detecting urolithiasis, virtually showing all stones, except in patients receiving indinavir. The accuracy of plain abdominal radiographs associated with ultrasound was similar to helical computed tomography, although the latter showed superiority as an isolated method. Data from literature suggest that whenever helical computed tomography is available, it is helpful in the evaluation of low back pain and also allows differential diagnosis between conditions that mimic the symptoms of urolithiasis.

Key words: Plain abdominal radiographs; Helical computed tomography; Intravenous pielography; Low back pain; Ultrasound of the urinary tract; Urolithiasis.

\section{INTRODUÇÃO}

A tomografia computadorizada (TC) helicoidal tem desempenhado papel importante no diagnóstico e tratamento da litíase renal, sendo nos últimos anos considerada, por alguns autores, como um "divisor de águas", papel antes atribuído à urografia excretora (UE) ${ }^{(\mathbf{1})}$. Outros autores têm sustentado que a radiografia simples

* Trabalho realizado no Serviço de Radiologia e Diagnóstico por Imagem do Hospital das Clínicas da Universidade Federal de Minas Gerais (HC-UFMG), Belo Horizonte, MG.

1. Médicos Residentes do Senviço de Radiologia e Diagnóstico por Imagem do HC-UFMG.

2. Médica Assistente do Serviço de Radiologia e Diagnóstico por Imagem, Coordenadora do Setor de Ultra-Sonografia do Instituto Alfa de Gastroenterologia do HC-UFMG.

Endereço para correspondência: Dr. Ricardo Miguel Costa de Freitas. Rua Santo Antônio do Monte, 386, Santo Antônio. Belo Horizonte, MG, 30330-220. E-mail: rmcfreitas@ig.com.br

Recebido para publicação em 3/9/2003. Aceito, após revisão, em 22/10/2003. do abdome associada à ultra-sonografia (US) são apropriadas na abordagem diagnóstica da dor lombar aguda e podem ser utilizadas como métodos alternativos quando recursos como a TC helicoidal são $\operatorname{limitados}^{(2)}$.

O objetivo deste trabalho é revisar a literatura sobre o tema, abordando as opções e as perspectivas diagnósticas. A literatura publicada nos últimos 30 anos comparando os métodos atualmente disponíveis para a avaliação de pacientes com dor lombar aguda foi revisada, com atenção aos seguintes métodos: a radiografia simples de abdome, a UE, a US e a TC helicoidal.

\section{DISCUSSÃO}

Os cálculos do trato urinário podem ser originários de placas de Randall situadas no revestimento dos ductos coletores nas papilas renais ${ }^{(3)}$, que podem se destacar e ser eliminadas ou formar cálculos, alojados freqüientemente na região das papilas e cálices. Os cálculos tendem a ser assintomáticos até causarem obstrução, com sintomas típicos de cólica renal ou ureteral ${ }^{(\mathbf{4})}$. Nos ureteres, o local mais comum de seu depósito é a junção uretero-vesical, ou acima desta, na porção pélvica do ureter ${ }^{(\mathbf{3})}$.

As composições mais comuns dos cálculos renais são o fosfato de cálcio, o oxalato de cálcio e o fosfato amoníaco magnesiano (estruvita). Os cálculos de cistina, urato e xantina são de baixa densidade e raros. Noventa por cento dos cálculos do trato urinário superior contêm cálcio suficiente para serem visualizados na radiografia simples de abdome ${ }^{(3)}$. No entanto, vários fatores seriam responsáveis pela baixa 
sensibilidade e especificidade da radiografia simples na detecção dos cálculos: estes podem ser obscurecidos por gases intestinais, sombras ósseas ou posicionamento incorreto do paciente, a magnificação geométrica pode distorcer o seu tamanho e a sua localização exata pode ser difícil de ser determinada. Além disso, a qualidade técnica da imagem também pode influenciar sua interpretação ${ }^{(5,6)}$. Ao se correlacionar os achados de radiografias simples do abdome com os da TC helicoidal (padrão ouro), alguns autores chegaram a afirmar que a radiografia simples do abdome teria sensibilidade de apenas 59\% para detectar cálculos urinários ${ }^{(4)}$.

A UE foi, por muito tempo, considerada o exame de imagem ideal na abordagem de pacientes com urolitíase. Porém, estudos atuais mostram que a sensibilidade, especificidade e acurácia diagnóstica da TC têmse tornado superiores à UE na avaliação desses pacientes. A TC é mais sensível para a detecção do cálculo (94\%), em relação à UE ( $52 \%$ a $69 \%$ ), e permite a determinação de seu tamanho e localização de forma precisa, além de fornecer diagnósticos alternativos para dor lombar aguda ${ }^{(7-9)}$. A UE permanece como o melhor método para a visualização do sistema coletor urinário e a TC como o melhor método para a detecção de cálculos urinários ${ }^{(\mathbf{1 0})}$.

A US vem sendo utilizada na detecção de cálculos renais desde $1961^{(11)}$ e se tornou um método de boa sensibilidade, inclusive para a detecção de cálculos não opacos, como os de ácido úrico ${ }^{(12,13)}$. Em pacientes submetidos a litotripsia extracorpórea por ondas de choque (LECO), a sensibilidade geral da US na detecção de cálculos foi de $96 \%$, sendo considerada superior à radiografia abdominal e levemente inferior à associação desta última com a nefroplanigrafia. De acordo com Middleton et al. ${ }^{(\mathbf{1 4})}$, ao randomizar pacientes pósLECO e pacientes que se submeteram a urografias por razões não relacionadas à nefrolitíase, a sensibilidade da US foi de $91 \%$. Os autores desse estudo afirmaram que a habilidade da US em detectar cálculos renais seria dependente do tamanho destes, mas independente da sua localização ou do tamanho do paciente ${ }^{(\mathbf{1 4})}$. A US também permite a detecção de outras condições como litíase biliar, pancreatite agu- da, apendicite aguda e massas abdôminopélvicas, que podem cursar com quadros dolorosos semelhantes à cólica renal ${ }^{(8)}$.

Levine et al. ${ }^{(4)}$ questionaram a sensibilidade da US de $96 \%$ para detectar cálculos renais ao se comparar com a radiografia abdominal e a tomografia convencional $^{(\mathbf{1 4})}$, e sugeriram ser esta sensibilidade substancialmente menor, dada a evidência de que a radiografia seria menos sensível do que previamente pensado. A TC helicoidal teria sensibilidade de $97 \%$ e especificidade de $96 \%$ para a detecção de cálculos urinários $^{(\mathbf{1})}$. A radiografia abdominal foi considerada mais sensível do que o topograma da TC ao revelar cálculos ureterais. Porém, alguns cálculos revelados pela TC helicoidal podem não ser vistos por nenhum dos dois métodos anteriores ${ }^{(\mathbf{1 5})}$.

O primeiro estudo comparando a sensibilidade da US para o cálculo ureteral em relação à TC helicoidal não contrastada concluiu ser esta última a melhor modalidade para detectar cálculos ureterais, com sensibilidade de $94 \%$ e especificidade de $97 \%$. Para a US estes valores foram de $19 \%$ e $97 \%$, respectivamente ${ }^{(\mathbf{5 , 1 6})}$.

Uma comparação prospectiva da US com a TC helicoidal para detecção de urolitíase $^{(17)}$ concluiu que a TC não contrastada tem sensibilidade maior para a detecção de cálculos ureterais do que a US. Foram estudados 23 pacientes com nefrolitíase, e a TC helicoidal detectou 22 casos (sensibilidade de 96\%), enquanto a US detectou 14 casos (sensibilidade de 61\%) $(\mathrm{p}=0,02)$. A especificidade de ambas as técnicas foi de $100 \%$.

Fowler et al. ${ }^{(\mathbf{1 8})}$ realizaram o primeiro estudo comparando a US com a TC helicoidal para avaliar cálculos dentro da pelve renal ou parênquima renal e atribuíram à primeira uma sensibilidade de $24 \%$ e especificidade de $90 \%$, concluindo ser a US de valor limitado para a detecção de cálculos renais. No entanto, esses autores admitiram ser o tamanho médio dos cálculos detectados à US de 7,1 mm e que $73 \%$ dos cálculos não visualizados à US apresentavam menos de $3 \mathrm{~mm}$ de diâmetro. $\mathrm{O}$ tamanho dos cálculos baseado nas medidas ultra-sonográficas e tomográficas foram concordantes em $79 \%$ dos casos. Naqueles discordantes, as dimensões diferiram em média $1,5 \mathrm{~mm}$.
A colimação do feixe da TC helicoidal a $3 \mathrm{~mm}$ é boa ao predizer o tamanho do cálculo e sua densidade: cálculos de ácido úrico apresentaram atenuação aproximada de 415 UH; de cistina, $668 \mathrm{UH}$; de estruvita, 919 UH; de oxalato de cálcio, 1.249 UH; de brucita, $1.383 \mathrm{UH}$; e de hidroxiapatita, $1.525 \mathrm{UH}$. Com o aumento da colimação, a capacidade de diferenciação dos cálculos é perdida ${ }^{(19)}$. Os cálculos radiotransparentes têm densidade de mais de $80 \mathrm{UH}$, sendo detectados pela $\mathrm{TC}^{(\mathbf{2 0})}$. No entanto, cálculos de indinavir, os quais são relativamente radiolucentes, não têm sido detectados $^{(19)}$.

Os sinais secundários de obstrução também podem ser detectados, como o aumento do volume renal, a presença de estratificação da gordura perirrenal, coleções líquidas perirrenais, hidronefrose, espessamento da fáscia renal e o sinal da borda tecidual. Este último, definido como uma borda circunferencial de atenuação de partes moles ao redor do cálculo renal, é um achado altamente específico para a presença de cálculo renal e para a diferenciação de um flebólito ${ }^{(\mathbf{3 , 8 , 2 1 - 2 3 )}}$.

À TC, a hidronefrose provavelmente reflete uma fase de obstrução subaguda à crônica, quando a pressão intraluminal normalizou e a peristalse diminuiu. Sabese que pacientes que se apresentam com duração de dor mais prolongada têm baixa taxa de passagem espontânea de cálcu$\operatorname{los}^{(21)}$. De acordo com estudos experimentais, o aumento da pressão no sistema coletor é considerado como a força propulsiva mais importante capaz de mover um cálculo a jusante do ureter, pelo menos durante a fase aguda da obstrução ${ }^{(24)}$.

Revendo esses sinais secundários à TC helicoidal, Takahashi et al. ${ }^{\text {(21) }}$ avaliaram 69 pacientes com cálculo ureteral único não localizado na junção uretero-pélvica. Trinta e cinco pacientes foram perdidos durante o seguimento; dos 34 pacientes restantes, 22 apresentaram eliminação espontânea do cálculo e 12 não responderam ao tratamento conservador. Comparando-se os dois últimos grupos, o diâmetro médio dos cálculos foi significativamente maior no segundo ( $\mathrm{p}=0,001)$, e apenas a estratificação da gordura perirrenal quanto à presença de coleções líquidas perirrenais foi significativamente maior no primeiro $(\mathrm{p}=0,044 \mathrm{e}$ 
$\mathrm{p}=0,021$, respectivamente). Cálculos com mais de $7 \mathrm{~mm}$ de diâmetro apresentaram 95\% de especificidade para a predição de insucesso terapêutico clínico, concluindose que os achados tomográficos compatíveis com cálculos menores e um alto grau de estratificação da gordura perirrenal ou quantidade aumentada de líquido perirrenal estariam associados com a passagem espontânea dos cálculos urinários. Grandes séries prospectivas seriam necessárias para confirmar esta hipótese.

Portanto, cálculos com dimensões menores teriam maior probabilidade de eliminação espontânea, o que, do ponto de vista prático, tornaria a avaliação imaginológica através de TC apropriada nos casos clinicamente significativos de obstrução do trato urinário.

De fato, Catalano et al. ${ }^{(2)}$ confirmaram os achados anteriores de que a TC helicoidal é uma ferramenta extremamente precisa na avaliação da dor lombar aguda. Comparando a TC com a US combinada com a radiografia convencional, evidenciou-se ser a TC significantemente superior no diagnóstico de ureterolitíase (sensibilidade de $92 \%$ versus $77 \%$ e valor preditivo negativo de $87 \%$ versus $68 \%$ ) e com acurácia semelhante no diagnóstico de causas alternativas de dor. No entanto, em seus resultados, esses autores concluíram ser a US combinada com a radiografia convencional uma alternativa valiosa em relação à TC em casos selecionados. Embora menos precisa do que a TC, esta combinação permitiu a detecção de todos os casos clinicamente relevantes, com consequiências semelhantes para o tratamento dos pacientes. Todos os pacientes que não foram diagnosticados à US/radiografia convencional recuperaram-se espontaneamente com o tratamento conservador, provavelmente por sofrerem cólica causada por cálculos de 1 a 3 mm. Assim, Catalano et al. ${ }^{(2)}$ sugeriram duas alternativas: 1) realizar a TC helicoidal nos pacientes com achados negativos à US/radiografia convencional; e/ou 2) proceder à TC apenas quando o clínico solicitante tenha forte convicção de que o paciente possa ter cólica acentuada ou uma causa relevante de dor não-ureterolitiásica (por exemplo, pacientes sem hematúria). Foi considerado que a TC deve ser empregada principalmente quando a avaliação inicial é duvidosa, tanto para obstrução ureteral quanto para causas alternativas ou quando a intervenção urológica é considerada necessária.

\section{OUTRAS MODALIDADES DIAGNÓSTICAS}

A TC por multidetectores ("multislice") tem sido recentemente apresentada como opção diagnóstica ${ }^{(25,26)}$. Tack et al. ${ }^{(27)}$ avaliaram 106 pacientes com suspeita de cólica renal por este método, sem a utilização de meio de contraste oral ou venoso. Destes, 38 (36\%) pacientes portavam cálculos ureterais, e a TC "multislice" apresentou sensibilidade, especificidade e acurácia diagnóstica variando entre $89,5 \%$ e $94,7 \%, 94,1 \%$ e $100 \%$, e $93,4 \%$ e $98,1 \%$, respectivamente. Estudos mais aprofundados são necessários para estabelecer a sua aplicabilidade prática.

\section{CONCLUSÃO}

A TC helicoidal tem sido considerada o método mais preciso no diagnóstico por imagem de cálculos do trato urinário, sendo defendida por alguns autores como o exame imaginológico de escolha na abordagem do paciente com dor lombar aguda em serviços de emergência ${ }^{(\mathbf{1})}$.

Por outro lado, outros autores têm proposto um algoritmo para a abordagem inicial destes pacientes, com a utilização da US associada à radiografia convencional inicialmente, devido à acurácia diagnóstica semelhante à da TC helicoidal nos casos clinicamente relevantes que não respondem ao tratamento conservador. Esta última seria recomendada em casos selecionados, reduzindo-se globalmente os gastos e a exposição à radiação, ao custo de um pequeno aumento no tempo necessário para se alcançar o diagnóstico ${ }^{(2)}$.

O método diagnóstico ideal pode ser considerado aquele de melhor custo-benefício, isto é, o de melhor resolutividade para o paciente e que alia o menor custo para o sistema de saúde. Considerando a associação da radiografia simples e US como métodos de boa sensibilidade na detecção dos casos de cálculos urinários clinicamente relevantes, o uso da TC helicoidal nesta situação pode ser reservado para aqueles em que o detalhamento anatômico será importante, como, por exemplo, na abordagem terapêutica invasiva.

\section{REFERÊNCIAS}

1. Smith RC, Varanelli M. Diagnosis and management of acute ureterolithiasis: CT is truth. AJR 2000; 175:3-6.

2. Catalano O, Nunziata A, Altei F, Siani A. Suspected ureteral colic: primary helical CT versus selective helical CT after unenhanced radiography and sonography. AJR 2002;178:379-87.

3. Lee FF Jr, Thornbury JR. O trato urinário. In: Juhl JH, Crummy AB, Kuhlman JE, eds. Paul \& Juhl Interpretação radiológica. 7ª ed. Rio de Janeiro: Guanabara-Koogan, 2000

4. Levine JA, Neitlich J, Verga M, Dalrymple N, Smith RC. Ureteral calculi in patients with flank pain: correlation of plain radiography with unenhanced helical CT. Radiology 1997;204:27-31.

5. Eray O, Çubuk MS, Oktay C, Yilmaz S, Çete Y, Ersoy FF. The efficacy of urinalysis, plain films, and spiral CT in ED patients with suspected renal colic. Am J Emerg Med 2003;21:152-4.

6. Narepalem N, Sundaram CP, Boridy IC, Yan Y, Heiken JP, Clayman RV. Comparison of helical computerized tomography and plain radiography for estimating urinary stone size. J Urol 2002;167: 1235-8.

7. Longo J, Akbar SA, Shaff T, Jafri ZH, Jackson RE. Prospective comparative study of non-contrast helical computed tomography and intravenous urogram for the assessment of the renal colic. Emerg Radiol 2001;8:285-92.

8. Dalla Palma L, Pozzi-Mucelli R, Stacul F. Presentday imaging of patients with renal colic. Eur Radiol 2001;11:4-17.

9. Vaswani KK, El-Dieb A, Vitellas KM, Bennett WF, Bova JG. Ureterolithiasis: classical and atypical findings on unenhanced helical computed tomography. Emerg Radiol 2002;9:60-6.

10. Dyer RB, Chen MYM, Zagoria RJ. Intravenous urography: technique and interpretation. RadioGraphics 2001;21:799-824.

11. Schlegel JU, Diggdon P, Cuellar J. The use of ultrasound for localizing renal calculi. J Urol 1961; 86:367-9.

12. Brennan RE, Curtis JA, Kurtz AB, Dalton JR. Use of tomography and ultrasound in the diagnosis of nonopaque renal calculi. JAMA 1980;244:594-6.

13. Pollack HM, Arger PH, Goldberg BB, Mulholland SG. Ultrasonic detection of nonopaque renal calculi. Radiology 1978;127:233-7.

14. Middleton WD, Dodds WJ, Lawson TL, Foley WD. Renal calculi: sensitivity for detection with US. Radiology 1988;167:239-44.

15. Assi Z, Platt JF, Francis IR, Cohan RH, Korobkin M. Sensitivity of CT scout radiography and abdominal radiography for revealing ureteral calculi on helical CT: implications for radiologic follow-up. AJR 2000;175:333-7.

16. Yilmaz S, Sindel T, Arslan G, et al. Renal colic: comparison of spiral CT, US and IVU in the detection of ureteral calculi. Eur Radiol 1998;8:212-7.

17. Sheafor DH, Hertzberg BS, Freed KS, et al. Nonenhanced helical CT and US in the emergency evaluation of patients with renal colic: prospective comparison. Radiology 2000;217:792-7.

18. Fowler KAB, Locken JA, Duchesne JH, William- 
son MR. US for detecting renal calculi with nonenhanced CT as a reference standard. Radiology 2002;222:109-13.

19. Saw KC, McAteer JA, Monga AG, Chua GT, Lingeman JE, Williams JC Jr. Helical CT of urinary calculi: effect of stone composition, stone size, and scan collimation. AJR 2000;175:329-32.

20. Segal AJ, Spataro RF, Linke CA, Frank IN, Rabinowitz R. Diagnosis of nonopaque calculi by computed tomography. Radiology 1978;129:447-50.

21. Takahashi N, Kawashima A, Ernst RD, et al. Ureterolithiasis: can clinical outcome be predicted with unenhanced helical CT? Radiology 1998;208:97102.
22. Etemad A, Brems-Dalgaard E, Thomsen HS. Outcome of intravenous urography in the year 2000. Abdom Imaging 2003;28:226-9.

23. Faintuch S, Goldman SM, Araujo MP, Kenney PJ, Ajzen SA, Szejnfed J. Obstructive ureterolithiasis: diagnostic value of renal attenuation measurements and comparison with other secondary signs on unenhanced helical CT. 88th Scientific Assembly and Annual Meeting of RSNA. Radiology suppl 2002:124.

24. Algood CB, Sood N, Fairchild T, Mayo ME. Experimental study of ureteral calculus disease: effects of calculus size, obstruction, and hydration. J Urol 1983;130:999-1004.
25. Caoili EM, Cohan RH, Korobkin M, et al. Urinary tract abnormalities: initial experience with multidetector row CT urography. Radiology 2002;222: 353-60.

26. Van Beers BE, Dechambre S, Hulcelle P, Materne $\mathrm{R}$, Jamart J. Value of multislice helical CT scans and maximum-intensity-projection images to improve detection of ureteral stones at abdominal radiography. AJR 2001;177:1117-21.

27. Tack D, Sourtzis S, Delpierre I, de Maertelaer V, Gevenois PA. Low-dose unenhanced multidetector CT of patients with suspected renal colic. AJR 2003;180:305-11. 